\title{
Diagnóstico de malformações congênitas: impactos sobre a saúde mental de gestantes
}

\author{
Diagnosis of congenital malformations: Impact \\ on the mental health of pregnant women
}

\author{
Ana Cristina Barros da CUNHA ${ }^{1}$ \\ José Paulo PEREIRA JUNIOR ${ }^{2}$ \\ Cláudia Lúcia Vargas CALDEIRA ${ }^{2}$ \\ Vanessa Miranda Santos de Paula CARNEIRO'
}

\begin{abstract}
Resumo
Malformação congênita, segunda maior causa de mortalidade infantil, constitui condição de vulnerabilidade importante na gravidez, que repercute desfavoravelmente na saúde mental da gestante. Objetivou-se estudar o impacto do momento do diagnóstico de malformação congênita sobre a saúde mental de 66 gestantes em atendimento pré-natal. Para isso, as participantes responderam ao Questionário "Momento da notícia", o qual identifica variáveis psicossociais relativas ao momento do diagnóstico da deficiência, e as Escalas Beck, para avaliar sinais e sintomas de ansiedade (Beck Anxiety Inventory) e depressão (Beck Depression Inventory). Todas receberam a notícia através de um médico, sendo 19 no segundo trimestre gestacional. Quatorze consideraram a transmissão do diagnóstico apropriada. No entanto, quando a notícia ocorreu no primeiro trimestre, tanto indicadores de ansiedade $(p=0,0009)$ quanto de depressão $(p=0,000004)$ se associaram ao momento da comunicação do diagnóstico; diferente de quando a gestante era comunicada no segundo trimestre, ao qual esteve associado somente os indicadores de depressão $(p=0,0462)$. Discute-se indicadores de ansiedade e depressão na gestação relacionados ao diagnóstico de malformação congênita como agravantes da vulnerabilidade física e psíquica durante a gestação.
\end{abstract}

Palavras-chave: Diagnóstico; Gestantes; Malformação congênita; Saúde mental.

\begin{abstract}
Congenital malformation, the second largest cause of infant mortality, is an important cause of vulnerability during pregnancy, and it has an unfavorable effect on the mental health of pregnant woman. The objective of this study was to investigate the impact of the moment of diagnosis of congenital malformation on the mental health of 66 pregnant women receiving prenatal care. The participants answered the questionnaire "Momento da notícia" (Moment of diagnosis), which identifies psychosocial variables related to the moment of diagnosis. The Beck Scales were also used to assess signs and symptoms of anxiety (Beck Anxiety Inventory) and depression (Beck Depression Inventory). All participants

$\nabla \nabla \nabla$

1 Universidade Federal do Rio de Janeiro, Instituto de Psicologia, Maternidade-Escola. R. das Laranjeiras, 180, Laranjeiras, 22240-003, Rio de Janeiro, RJ, Brasil. Correspondência para/Correspondence to: A.C.B. CUNHA. E-mail: <acbcunha@yahoo.com.br>.

2 Universidade Federal do Rio de Janeiro, Maternidade-Escola. Rio de Janeiro, RJ, Brasil. Apoio: Fundação de Amparo à Pesquisa do Estado do Rio de Janeiro (Processo nº E-110.660/2013).
\end{abstract}


were given the diagnosis by a physician, and 19 were notified during the second trimester of pregnancy. The diagnosis communication was considered adequate by fourteen participants. However, when the diagnosis was notified during the first trimester of pregnancy it was associated with indicators of anxiety $(p=0.0009)$ and depression $(p=0.000004)$. Different results were found when the mother was notified during the second trimester, when only the indicators of depression were associated to the diagnosis received $(p=0.0462)$. The present study addressed the indicators of anxiety and depression during pregnancy associated to the diagnosis of congenital malformation as risk factors for the physical and psychological vulnerability during this period.

Keywords: Diagnosis; Pregnant women; Malformations, congenital; Mental health.

Considerando a gravidez como um fenômeno complexo que envolve mudanças de diversas ordens (Vasconcelos \& Petean, 2009), a gestação configura-se como um período de grande vulnerabilidade tanto física, em virtude das mudanças biofisiológicas decorrentes, quanto psíquica, por conta da fragilidade emocional resultante da ambivalência de sentimentos, característica desse momento e que merece atenção especial (Sarmento \& Setúbal, 2003; Setúbal, Barini, Zaccaria, \& Silva, 2004; Roecker, Mai, Baggio, Mazzola, \& Marcon, 2012). Desde o momento da fecundação até o nascimento, tanto a gestante quanto o bebê passam por experiências psicológicas, fisiológicas e sociais únicas, as quais fazem com que a mulher crie várias expectativas em relação ao novo ser que está por parir e o papel o qual deverá assumir. Isso pode resultar em um processo de super idealização da maternidade, de tal maneira que pode gerar sintomas psicológicos os quais transformam esse momento em um período particularmente estressante e propenso a crises (Leithner, 2011; Perosa, Canavez, Silveira, Padovani, \& Peraçoli, 2009; Rosa et al., 2010; Setúbal et al., 2004), sobretudo quando ocorrem complicações ao longo da gravidez.

Obviamente, o desejo de todo casal é ter um filho saudável e perfeito, de acordo com expectativas pessoais, sociais e culturais (Vasconcelos \& Petean, 2009) e que traga momentos de alegria e felicidade (Perosa et al., 2009). Um processo de projeção ocorre naturalmente durante a gravidez, gerando o desejo por um filho idealizado, um "bebê ideal" (Gomes \& Piccinini, 2010). No entanto, quando acontece desse bebê idealizado ser substituído por um real, com um diagnóstico também real de malformação congênita, a gravidez, período já marcado por fantasias angustiantes em 602 relação à integridade do feto (Leithner, 2011; Soulé,
1992), se transforma em um momento de intenso sofrimento e angústia emocional. Tudo isso ocorre devido à perda da possibilidade da gestação ideal (Barros, Santos, Lima, Fonseca, \& Lovisi, 2013) e ao aniquilamento de expectativas e sonhos, os quais deverão ser refeitos à luz de uma nova realidade, sempre de difícil adaptação (Antunes \& Patrocínio, 2007).

O enfrentamento da situação requer um processo de adaptação que irá depender de vários fatores, os quais influenciarão na capacidade de manejo desse diagnóstico. Além da gravidade da malformação, da estrutura emocional do casal e da dinâmica familiar, a disponibilidade de informação e uma atenção especializada da rede de saúde são fundamentais para o casal e sua família se reorganizarem diante do diagnóstico de malformação (Setúbal et al., 2004). Dessa forma, destaca-se a importância da atuação dos profissionais de saúde, que poderão ajudar a família a identificar tanto os seus recursos como suas fragilidades e necessidades, fornecendo meios para lidar com a situação (Sunelaitis, Arruda, \& Marcon, 2007).

Diante da vivência dessa condição, tanto a mãe quanto a família necessitarão, por parte dos profissionais de saúde, de atenção e cuidados especiais a fim de que se sintam respeitados e apoiados, desde o pré-natal até o puerpério (Roecker et al., 2012). Segundo Ha, Anat e Longnecker (2010), dentre esses cuidados destaca-se uma relação médico-paciente pautada em habilidades interpessoais e de comunicação do médico, o qual deve ser capaz de reunir as informações necessárias para um diagnóstico preciso, além de aconselhar de forma adequada com instruções terapêuticas claras, priorizando uma relação acolhedora com o paciente.

A relação médico-paciente constitui grande desafio quando se trata da comunicação da malfor- 
mação congênita (Loaiza \& Arroyave, 2009). A literatura indica que os médicos se sentem apreensivos no desafio de transmitir más notícias por não terem treinamento suficiente em habilidades de comunicação interpessoal durante sua formação (Ha et al., 2010; Loaiza \& Arroyave, 2009; Perosa \& Ranzani, 2008). Dessa forma, deixam de cumprir seu papel de facilitar a adesão ao tratamento e amenizar o sofrimento associado ao diagnóstico (Perosa \& Ranzani, 2008).

Loaiza e Arroyave (2009) consideram que a má noticia deve ser comunicada com base em alguns princípios os quais pressupõem desde o preparo do profissional, que deve estar familiarizado com as informações clínicas relevantes, até a escolha de um local para comunicação onde exista o mínimo de interrupções. É fundamental que a transmissão de informações sobre o diagnóstico ocorra de forma compreensível ao paciente, com oferecimento de uma esperança realista sobre o prognóstico da situação e de um suporte emocional para o enfrentamento, sendo este último também indicado por Leithner (2011). Dessa forma, estudos sobre a comunicação da notícia do diagnóstico de malformações congênitas e seus impactos são fundamentais, ainda mais quando se sabe que a incidência dos defeitos congênitos é de três para cada 100 nascimentos e de uma em cada quatro mortes fetais durante a gravidez ou na primeira semana de vida (Aymé, 2005), com prevalência de 22985 casos para 2913160 nascidos vivos (Brasil, 2013a).

Atualmente, através de exames como a ultrassonografia, os pais tomam conhecimento da malformação do seu bebê precocemente ao longo da gestação. Logo, a medicina fetal pode exercer um duplo papel no enfretamento da situação tanto acalmando quanto perturbando ainda mais a reorganização do casal (Antunes \& Patrocínio, 2007). Além disso, o diagnóstico precoce pode antecipar as angústias maternas (Sousa, 2003), desencadeando sentimentos de dor, desespero e culpa, os quais também podem causar sequelas psicológicas graves a longo prazo (Leithner, 2011; Loaiza \& Arroyave, 2009; Perosa et al., 2009). Isso porque o impacto de um feto malformado ou deficiente pode tornar- se muito angustiante e desestruturante para o casal e sua família (Vasconcelos \& Petean, 2009).

Essa situação é digna de atenção, tendo em vista que na gestação, sendo um período sensível por si só, pode ocorrer maior estresse e ansiedade parental diante desse tipo de notícia, os quais são vivenciados ao longo de todo processo de investigação diagnóstica (Loaiza \& Arroyave, 2009; Setúbal et al., 2004; Sousa, 2003). Isso resulta em rápido desinvestimento na gravidez, com consequências na saúde mental da mulher e repercussões na dinâmica familiar. Dessa forma, é fundamental a realização de estudos que possam contribuir com dados empíricos os quais auxiliem na compreensão dessa vivência pelo casal grávido.

Esse tema encerra ainda relevância social, já que, em casos de malformações incompatíveis com a vida, esses pais ainda têm de lidar com um dilema moral: a difícil decisão de interromper ou não o curso da gravidez (Antunes \& Patrocínio, 2007; Leithner, 2011). Essa decisão é permitida por lei no Brasil nos casos de anencefalia (Brasil, 2013b). Além disso, o casal precisa lidar com o estigma de "defeituosos", "imperfeitos" ou "anormais", assim como potenciais transmissores de defeitos às próximas gerações (Cardoso, 2001).

Logo, um diagnóstico bem executado e esclarecidamente comunicado pode servir para que o casal e toda a família possa enfrentar de forma mais resiliente a situação, a partir da prática de um cuidado humanizado por toda a equipe de saúde. O cuidado humanizado certamente ajudará na elaboração do luto vivenciado pelos pais logo após as reações iniciais ao diagnóstico de malformação, auxiliando-os a lidar com os sentimentos ambivalentes de esperança e medo, sofrimento e confiança, otimismo e pessimismo resultantes das diferentes expectativas positivas e negativas criadas por uma gestação de risco (Chaplin, Schwitzer, \& Perkoulidis, 2005).

No entanto, ainda que a literatura demonstre que essas ambivalências, junto com reações de irritabilidade e isolamento, ocorram logo após uma gestação traumatizante e mais precisamente em seu período final (Chaplin et al., 2005), não foram encontrados estudos que avaliem o impacto da notí- 
cia da malformação congênita nos diferentes períodos da gestação, o que motivou nosso estudo.

Ressalta-se, assim, a importância de se estudar o impacto desse fator de risco ao longo da gestação, discutindo possíveis desdobramentos em termos psíquicos, os quais podem ir além do próprio bem-estar físico (Gorayeb, 2008) ou mesmo variar em relação ao período gestacional, de acordo com as ansiedades típicas de cada trimestre (Sarmento \& Setúbal, 2003). Diante da relevância científica e social de estudos sobre essa temática, o objetivo da presente pesquisa foi investigar o impacto do momento do diagnóstico de malformações congênitas sobre a saúde mental materna com base na análise de indicadores emocionais (ansiedade e depressão) e da percepção da notícia desse diagnóstico por gestantes de risco.

\section{Método}

\section{Participantes}

Participaram desta pesquisa de delineamento descritivo quantitativo com amostra de conveniência, 66 gestantes com média de 28 anos de idade e diagnóstico confirmado de malformação congênita. Foram incluídas na amostra toda e qualquer mulher grávida atendida no período de 2010 a 2012 pelo Setor de Medicina Fetal de uma maternidade pública de ensino da cidade do Rio de Janeiro, cujo serviço é especializado na investigação diagnóstica e atendimento pré-natal à gestação de alto risco. Cabe esclarecer que esse Setor de Medicina Fetal serve como referência no sistema público de saúde da cidade do Rio de Janeiro para encaminhamento de casos para investigação diagnóstica, ou seja, gestantes com suspeita de malformação congênita são encaminhadas para confirmação diagnóstica e posterior seguimento do pré-natal em sua unidade hospitalar de origem.

\section{Instrumentos}

Foram utilizados os seguintes instrumentos

604 para coleta de dados, na seguinte ordem de apli- cação: 1) Protocolo de dados gerais, para identificação das variáveis psicossociais pessoais e familiares; 2) Escala Beck (BAl, Beck Anxiety Inventoryl Inventário Beck de Ansiedade) e depressão (BDI, Beck Depression Inventory/Inventário Beck de Depressão), para avaliação psicológica de sinais e sintomas de ansiedade e depressão diante da notícia do diagnóstico de malformação congênita na gravidez; e 3) Questionário "Momento da notícia", para identificação das variáveis psicossociais relativas ao momento do diagnóstico de malformação congênita.

Este último é um instrumento adaptado de Ramos, Hoffmann e Regen (1985) e tem por objetivo avaliar o momento em que a mulher recebeu a notícia do diagnóstico do bebê, mais especificamente como a notícia foi comunicada e quais as primeiras reações ocorridas, bem como possíveis estratégias de enfrentamento utilizadas neste contexto. É um instrumento com perguntas abertas e fechadas aplicado em entrevista individual com a participante.

\section{Procedimentos}

Em consulta médica no setor mencionado, as gestantes eram convidadas a participar da pesquisa, momento no qual assinavam o Termo de Consentimento Livre e Esclarecido aprovado pelo Comitê de Ética da própria maternidade (Processo $n^{\circ}$ 0033.0.361.361-9, de 28 de junho de 2010). Logo após saírem da consulta em que receberam a notícia ou confirmaram a suspeita diagnóstica inicial, as gestantes respondiam aos instrumentos acima mencionados. Cabe ressaltar que o Questionário "Momento da notícia" foi aplicado em entrevista individual e respondido por apenas 31 gestantes do total de 66 , uma vez que a coleta de dados, posterior à consulta de confirmação do diagnóstico, não era completa em alguns casos, pois a gestante não tinha tempo de concluir a aplicação e não retornava à instituição, por se tratar de um caso de investigação diagnóstica.

As Escalas Beck foram administradas para avaliar indicadores de ansiedade e depressão nas gestantes diante da sua condição de vulnerabilidade, isto é, o diagnóstico de malformação congênita do seu bebê. Para tanto, foram aplicados 
dois inventários dessa escala, os quais têm normas e padronização brasileiras com comprovada propriedade psicométrica (Cunha, 2001): o Inventário $\mathrm{BAI}$ e o BDI. O primeiro mede a intensidade de sinais e sintomas de ansiedade em quatro critérios de classificação: 1) absolutamente não; 2) levemente: não me incomodou muito; 3) moderadamente: foi muito desagradável, mas pude suportar; e 4) gravemente: dificilmente pude suportar. Já o segundo mede a intensidade da depressão classificada em quatro níveis crescentes de gravidade de sintomas depressivos: 0: mínimo, 1: leve, 2: moderado e 3: grave. Para ambos os inventários foi adotada a classificação da versão de 2001 do Manual das Escalas Beck (Cunha, 2001) que propõem a seguinte pontuação: (a) para o BAl: mínimo: 1 a 7; levemente: 8 a 15; moderadamente: 16 a 25; gravemente: 26 a 63; e (b) para o BDI: mínimo: 1 a 9; leve: 10 a 16; moderado: 17 a 29; grave: 30 a 63 .

Todas as participantes foram avaliadas pelas Escalas Beck. Para análise do Questionário "Momento da notícia", foi feito um recorte descritivo por ter sido aplicado a somente 31 gestantes. Os dados da Escala Beck foram processados e analisados de acordo com os parâmetros de correção do manual e tratados estatisticamente no software BioEstat 5.0, quando também foi aplicado o teste Qui-quadrado para verificar associações entre os indicadores emocionais (ansiedade ou depressão) e o momento da gestação em que a notícia da malformação era comunicada.

O relato verbal coletado pelo Questionário "Momento da notícia" foi analisado pelo método de análise de conteúdo de Bardin (1977), tendo sido registrado e posteriormente analisado em termos de frequência de ocorrência das seguintes categorias: (a) momento da notícia: "quem deu a notícia" (médico; outros); "quando recebeu a notícia" (1, $2^{\circ}$ ou $3^{\circ}$ trimestre de gestação); (b) reação pessoal à notícia: "como se sentiu" (sentimentos negativos; positivos; ambivalentes); "como se sente atualmente" (sentimentos negativos; positivos; ambivalentes); "o que achou de como foi informada" (adequado; inadequado); "como deveria ser dada a notícia" (igual; melhor); "como reagiu à notícia" (atitude positiva; negativa); e (c) reação familiar à notícia: "como o pai reagiu à notícia" (atitude positiva; negativa); " mudanças na relação familiar após a notícia" (avaliação negativa; positiva).

\section{Resultados}

Pela análise do protocolo de dados gerais, foi possível verificar que, em geral, as gestantes, que tinham média de idade de 28 anos, contavam com companheiro ( $n=62 ; 94 \%)$, em relacionamento estável por período que variou de oito meses a 21 anos. Do total, 56\% ( $n=37)$ trabalhava fora e $80 \%(n=53)$ declarou contar com suporte social familiar para ajudá-las durante a gestação. Quanto ao nível de escolaridade, 45\% ( $n=30)$ tinha Ensino Médio completo, $42 \%(n=28)$, Ensino Fundamental e $12 \%(n=8)$, Ensino Superior completo.

Os dados do Questionário "Momento da notícia" revelaram que todas as gestantes foram informadas por um médico obstetra ou ginecologista (100\%: $n=31$ ); sendo que isso aconteceu, em $61 \%$ dos casos ( $n=19)$, no segundo trimestre de gestação. A forma como foi transmitida a notícia do diagnóstico foi percebida por $55 \%(n=17)$ das gestantes como adequada. Cuidado na confirmação do diagnóstico, postura atenciosa e explicações voltadas para perspectivas futuras foram ressaltados por $55 \%$ das entrevistadas, como pode ser observado nos relatos: (a) "A médica super atenciosa foi bem cautelosa, onde a mesma não deu 100\% de algo sério e sim que iriam ser feitos diagnósticos"; (b) "Falaram de maneira carinhosa para acalmar"; e (c) "Explicou que não era cisto [o que havia sido diagnosticado na primeira ultrassonografia realizada fora da instituição] e disse que o bebê iria andar e falar".

No entanto, $45 \%(n=14)$ das gestantes considerou inadequada a maneira como o médico transmitiu o diagnóstico. Essas avaliações negativas pautaram-se principalmente no excesso de objetividade, como ilustra o seguinte relato: "Fez exame e confirmou a Translucência Nucal alterada e disse que geralmente morre intraútero". As gestantes ressaltaram, ainda, aspectos relativos à forma como foi transmitida a notícia, utilizando a expressão "Nua e crua". 
Conforme esperado, nenhuma gestante relatou sentimentos positivos relacionados ao diagnóstico de malformação congênita. Além de tristeza, houve declarações sobre desespero ("Triste, desesperada, sem ter certeza de nada") e autopiedade ("Tristeza, incapacidade, porque isso foi acontecer comigo?"). Na reação frente ao diagnóstico, em geral, as gestantes relataram atitudes negativas, como choque ("Não caiu a ficha, meio em choque"), ainda que confiança e tranquilidade tenham sido reações posteriores, características dos casos de investigação e confirmação do diagnóstico.

Setenta e quatro por cento dos companheiros/pais das crianças também tiveram uma reação negativa ao diagnóstico ("Ficou preocupado, nervoso e triste"). Somente $24 \%$ das gestantes perceberam essa atitude como tendo repercussão negativa no casamento, com posterior aproximação do casal ("A relação mudou para melhor, com mais união e sabedoria para lidarmos com a situação atual"), principalmente nos casos de investigação e confirmação diagnósticas. No caso da família, foram mais comuns atitudes negativas, com reação de choque e surpresa em $82 \%$ dos casos.

No que se refere à análise dos indicadores emocionais de ansiedade e depressão diante do diagnóstico, na Figura 1 pode ser observado que todas as gestantes apresentaram ansiedade e 78\% ( $n=50$ ) tiveram depressão, com predominância de sinais leves (38\%: $n=25$ e $62 \%: n=41$, respectivamente). O número de gestantes com sinais de ansiedade moderada ( $23 \%: n=15)$ também merece destaque, ainda que semelhante àquelas com sinais mínimos (26\%: $n=17)$. No que se refere à depressão, cabe destacar que, ainda que $24 \%(n=16)$ não tenha apresentado sinais de depressão, houve predominância de gestantes com sinais leves (62\%: $n=41)$ e mesmo severos da patologia $(10 \%: n=7)$.

Analisou-se, também, a incidência de ansiedade e depressão em relação ao momento da gestação em que ocorreu o recebimento da notícia de malformação congênita (Figura 2). Observou-se que, das 31 gestantes as quais responderam ao Questionário "Momento da notícia" e foram avaliadas pelas Escalas Beck, 77,3\% ( $n=24)$ apresentava indicadores de ansiedade e 83,8\% ( $n=26)$, de depressão. Ao analisar ambas as patologias relacionadas ao momento da gestação em que foi transmitido o diagnóstico, verificou-se que houve maior ocorrência de ambos os indicadores emocionais no segundo trimestre, com frequência de $45,1 \%(n=14)$ de gestantes apresentando indicadores de ansiedade e $54,8 \%(n=17)$, de depressão. Vale ressaltar que, enquanto no primeiro trimestre gestacional a frequência de gestantes com indicadores de depressão foi maior $(19,3 \%: n=6)$ do que daquelas com ansiedade (16,1\%: $n=5)$, no terceiro trimestre ansiedade e depressão foram equivalentes $(12,9 \%$ : $n=4)$. Com o intuito de verificar associações entre os indicadores emocionais de ansiedade e depressão e os diferentes períodos gestacionais em que a comunicação da notícia de malformação ocorreu, foi feita análise estatística inferencial pelo teste Qui-quadrado, adotando-se $p<0,05$ como índice de significância.

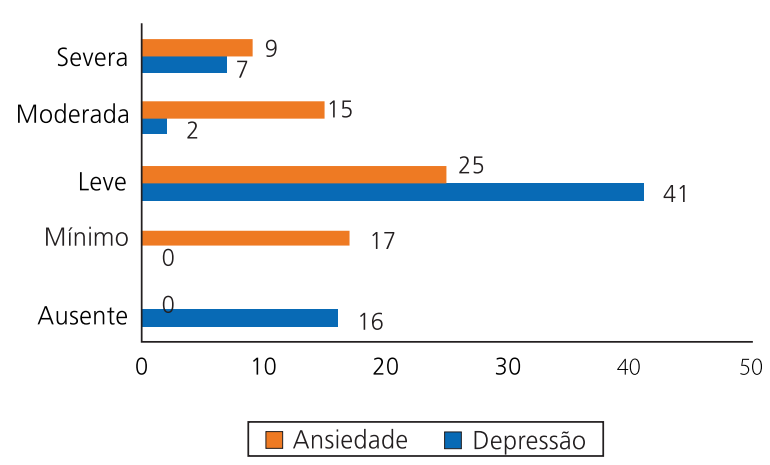

Figura 1. Níveis dos indicadores emocionais de ansiedade e depressão nas gestantes $(n=66)$.

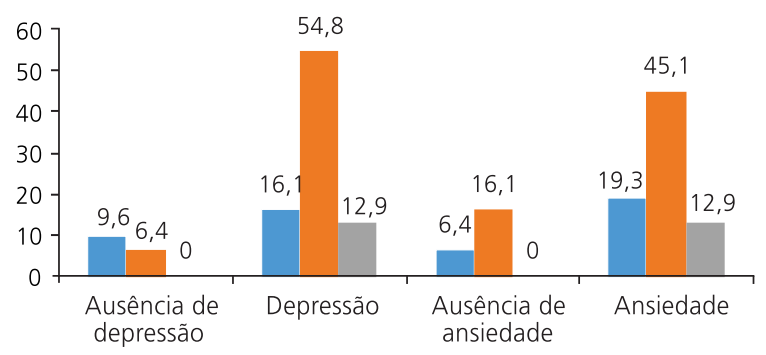

$\square$ Primeiro trimestre $\square$ Segundo trimestre $\square$ Terceiro trimestre

Figura 2. Ausência ou presença de ansiedade e depressão de acordo com o período gestacional em que ocorreu o diagnóstico $(n=31)$.

Nota: Indicadores emocionais x Momento da notícia $(n=31)$. 
Quando a notícia foi recebida no primeiro trimestre, houve ocorrência em níveis significativos tanto de indicadores de ansiedade quanto de depressão $\left(\chi^{2}=10,95 ; p=0,0009\right.$; e $\chi^{2}=21,10$; $p=0,000004$, respectivamente), o que significa que ambos os indicadores emocionais estiveram presentes desde o início da gestação. Por outro lado, quando a gestante era comunicada no segundo trimestre, foi significativa a frequência de indicadores de depressão $\left(\chi^{2}=3,97 ; p=0,0462\right)$ sem, no entanto, ocorrer associação significativa com ansiedade $\left(\chi^{2}=0,282 ; p=0,5955\right)$. Já no terceiro trimestre, a comunicação do diagnóstico não se associou significativamente com nenhum dos indicadores emocionais investigados (Tabela 1).

Tabela 1

Associações entre indicadores de ansiedade e depressão e os momentos da comunicação do diagnóstico durante a gestação

\begin{tabular}{lcc}
\hline \multirow{2}{*}{ Momento da notícia do diagnóstico } & \multicolumn{2}{c}{$p$} \\
\cline { 2 - 3 } & Ansiedade & Depressão \\
\hline $1^{\circ}$ trimestre & 0,0009 & 0,000004 \\
$2^{\circ}$ trimestre & 0,5955 & 0,0462 \\
$3^{\circ}$ trimestre & $-{ }^{*}$ & $-{ }^{*}$ \\
\hline
\end{tabular}

Nota: *Frequência inferior a 5; $p \leq 0.05$.

\section{Discussão}

De acordo com os resultados, confirma-se que o diagnóstico de malformação congênita mobiliza variáveis psicoafetivas relacionadas ao enfrentamento da situação diagnóstica. Além disso, constitui condição potencialmente desfavorável à saúde mental materna, como pôde ser observado devido à ocorrência de indicadores de depressão e ansiedade na amostra estudada, com prevalência deste último, bem como uma associação estatisticamente significativa de ambos os indicadores no primeiro trimestre e associação significativa de depressão quando o diagnóstico era comunicado no segundo trimestre gestacional. Isso ressalta a importância da adoção de medidas de proteção e de promoção do desenvolvimento e saúde materno-infantil, como, por exemplo, um manejo adequado da equipe de saúde no momento de dar a notícia do diagnóstico de malformação.
Nota-se que o momento do diagnóstico é extremamente delicado tanto para a gestante e sua família quanto para o profissional, que deve buscar adotar uma postura empática, oferecendo, além de informações claras e compreensíveis ao paciente, um suporte emocional o qual respeite a vivência da dor e angústia resultante da incerteza do futuro do filho (Ha et al., 2010; Leitner, 2011; Loaiza \& Arroyave, 2009). Isso foi constatado em alguns casos relatados por participantes do estudo: "Normal, mas receber a notícia é ruim. Os médicos também ficam tristes. Fui bem atendida".

Referente à relação médico-paciente, é válido ressaltar a dificuldade do médico em transmitir más notícias de maneira realista, sem provocar o desinvestimento emocional da gestante no bebê ou transmitir seus valores pessoais (Ha et al., 2010). Particularmente em casos de inviabilidade do feto, quando os médicos têm que se deparar com a morte fetal, é comum terem dificuldade de lidar com suas próprias emoções, se sentindo despreparados para acolher e dar suporte aos pacientes (Ha et al., 2010; Loaiza \& Arroyave, 2009; Montero et al., 2011).

Esse fato foi confirmado pelas gestantes participantes do estudo quando expressaram o direito de manter e conduzir a gestação, independentemente da inviabilidade da mesma ("Esperançosa. Eu quero que venha do jeito que for"). Essa situação pode ser particularmente difícil para o médico, o qual nem sempre consegue ter a postura acolhedora aconselhável para a situação (Loaiza \& Arroyave, 2009), conforme é possível observar nesse relato: "Desumano. Ele disse que não tinha feto, que não adiantava lutar por nada". Para esquivar-se do confronto emocional, o profissional foca apenas nos aspectos biológicos durante o contato com a paciente, se comportando de maneira distante e negando, por vezes, a gravidade da situação para a mulher (Montero et al., 2011). Esse tipo de comunicação médica pode se relacionar à condição emocional desfavorável, como a depressão observada nas gestantes do segundo trimestre do estudo, o que pode agravar a vulnerabilidade psíquica da gestante.

No entanto, é sabido que essas mulheres investem em um luto pela perda do filho idealizado, 
que pode se desdobrar em variadas reações, desde as iniciais, ambivalentes (sentimentos de esperança e medo, sofrimento e confiança, otimismo e pessimismo), até irritabilidade e isolamento. Todas são formas de elaborar o impacto inicial de uma gestação traumatizante (Chaplin et al., 2005). Posteriormente, esse luto dá lugar a um processo de adaptação o qual, ainda que com dor e sofrimento, repercute na aceitação da gravidez, fato observado no relato das gestantes do presente estudo. As participantes afirmaram terem conseguido, aos poucos, reelaborar o significado do diagnóstico, sentindo-se mais confiantes e tranquilas, como observado a seguir: (a) "Preocupada, porém confiante" e (b) "Mais tranquila, pois é um quadro tratável ou até regressivel".

Dessa forma, os dados de ansiedade e depressão, com frequência estatisticamente significativa no primeiro trimestre da gestação, podem ser considerados indicadores, mas não determinantes de um transtorno psicológico posterior. Corrobora essa afirmativa o fato de ter ocorrido, ao longo do estudo, a ressignificação do diagnóstico pelas gestantes sob investigação, as quais receberam a confirmação da malformação posteriormente e cujas repercussões na dinâmica familiar resultaram em maior proximidade com o marido e a família. Estes passaram de uma reação inicial de choque e surpresa para o acolhimento com suporte social e emocional, o que foi comum à maioria daquelas gestantes. Essa aproximação do casal ocorreu em momento posterior à reação negativa do companheiro e proporcionou o fortalecimento pessoal através, por exemplo, da fé, como destaca o relato: "Triste, mas ao mesmo tempo muito confiante que tudo pode acontecer para quem tem fé".

Considerando que a mulher é aquela que "carrega o bebê", ela estará mais propensa a vivenciar sentimentos de culpa, considerando-se responsável pelo desfecho da gravidez, sobretudo quando o diagnóstico ainda é inconclusivo (Sousa \& Muza, 2011). Para Sousa e Muza (2011), o pai, nessa situação, acaba por apresentar uma tendência a responder de forma mais controlada e com menos sinais externos, por sentir a necessidade de "ser forte" e "tomar conta" da esposa, o que foi obser- vado como reação posterior: "Não transpareceu desespero e sim força para que eu confiasse que tudo iria dar certo!".

Da mesma maneira, é frequente o desenvolvimento de ansiedade e/ou depressão antes mesmo do bebê nascer. De acordo com Farias e Villwock (2010), a ansiedade e a angústia vivenciadas pela mulher são recorrentes nos diferentes períodos gestacionais, quando ocorrem perdas e ganhos psíquicos e físicos. Assim, a presença dos indicadores de ansiedade e depressão nos dois primeiros trimestres de gestação podem estar associados tanto à superidealização da maternidade, que pode repercutir em sintomas psicológicos os quais transformam a gravidez em momento especialmente estressante e propenso a crises (Leithner, 2011; Perosa et al., 2009; Rosa et al., 2010; Setúbal et al., 2004), quanto à perda da possibilidade da gestação ideal (Barros et al., 2013; Gorayeb, 2008) e das expectativas e sonhos construídos acerca da gravidez (Antunes \& Patrocínio, 2007).

Cabe ressaltar que, quando a gestante recebia a notícia no segundo trimestre, o nível de indicadores de depressão era significativa $(p=0,0462)$ em comparação com o de ansiedade $(p=0,5955)$. Esse dado é contrário ao que pode ser encontrado na literatura, e surgiu, possivelmente, porque a gestante e sua família estavam, nesse período, atualizando as expectativas em relação ao bebê, o qual, com o avanço da gestação e a proximidade do parto, estava cada vez mais distante do bebê imaginado. Assim, a cada ultrassonografia realizada é confirmado novamente o diagnóstico de malformação e, por causa disso, a gestante fica cada vez mais distante do sonho do filho ideal. Diante disso, é possível que a esperança de ocorrer um "milagre" (Chaplin et al., 2005), vivenciada por gestantes do primeiro trimestre, tenha se transformado em desesperança nas do segundo, o que facilitou a associação com indicadores de depressão e de ansiedade.

Outro dado que também pode ter corroborado para ocorrência dos indicadores de ansiedade e depressão no segundo trimestre diz respeito à percepção negativa da comunicação do diagnóstico por $45 \%$ das gestantes. Isso confirma a importância 
da formação médica para comunicação de diagnósticos difíceis, assim como as dificuldades de ordem emocional que o profissional enfrenta quando lida com situações como essa em sua rotina profissional (Ha et al., 2010; Loaiza \& Arroyave, 2009; Montero et al., 2011).

As gestantes participantes destacaram os aspectos negativos em relação à interação com o médico no momento da comunicação do diagnóstico, algumas, inclusive, criticaram a postura pouco acolhedora que o profissional assumiu, como exemplifica esse relato: "Disse que geralmente morre intraútero". Com certeza essa postura em nada facilita a adesão ao tratamento e a minimização do sofrimento associado ao diagnóstico (Perosa \& Ranzani, 2008), ainda mais considerando que, nesses períodos, o casal grávido pode receber um diagnóstico mais preciso e próximo da real condição clínica de malformação do filho, o que nem sempre é possível no primeiro trimestre quando se está em fase de investigação inicial.

Dessa forma, é extremamente importante para a elaboração do luto diante do diagnóstico de malformação congênita que a relação médico-paciente seja pautada nos princípios indicados na literatura (Ha et al., 2010; Leitner, 2011; Loaiza \& Arroyave, 2009). No entanto, não se pode olvidar que nem sempre o médico está devidamente capacitado ou se sente apto para a comunicação de más notícias (Ha et al., 2010), sendo crucial prepará-lo para isso, de modo a facilitar a adesão, amenizar o sofrimento associado à malformação e aumentar a compreensão da informação transmitida (Perosa \& Ranzani, 2008).

Cabe ressaltar que a comunicação do diagnóstico no terceiro trimestre não teve associação estatisticamente significativa com nenhum dos indicadores emocionais investigados. No entanto, ainda assim houve ocorrência de depressão e ansiedade em todas as gestantes as quais receberam o diagnóstico naquele período. Isso é particularmente importante de ser discutido neste artigo, ainda que de forma restrita, uma vez que não foi possível compor uma amostra com número equivalente de participantes em cada trimestre gestacional. Esse dado demonstra ser fundamental o acompanhamento da saúde mental das gestantes com diagnóstico de malformação, sobretudo no final da gravidez, quando são caraterísticos, além do medo do parto, questionamentos sobre sua competência em "maternar".

Outra limitação do estudo diz respeito à amostra estudada, que foi composta tanto por mulheres que recebiam pela primeira vez o diagnóstico quanto por aquelas as quais chegavam ao serviço com uma suspeita de malformação a ser investigada. Ainda que a literatura indique ser forte o impacto emocional inicial e que, portanto, uma avaliação psicológica mais fidedigna deveria ser feita em outro momento, por limitações metodológicas a entrevista teve que ocorrer logo após a notícia inicial ou confirmação da malformação. Assim, não foi possível controlar essa variável, por ter sido adotada a amostragem por conveniência e o delineamento de estudo exploratório, cujo critério único de inclusão foi ter diagnóstico de malformação confirmado.

De todo modo, a literatura indica que em qualquer um dos casos esse tipo de diagnóstico ocasiona intenso sofrimento psíquico o qual pode, inclusive, resultar em problemas psicológicos graves a longo prazo (Leithner, 2011; Loaiza \& Arroyave, 2009; Perosa et al., 2009). Essa condição de vulnerabilidade psíquica vai além do próprio bem-estar físico (Gorayeb, 2008), porque configura-se na quebra dos desejos e expectativas parentais, além de ameaça à dinâmica familiar (Antunes \& Patrocínio, 2007). Logo, deve-se ter atenção à saúde mental da gestante com diagnóstico de malformações congênitas em todo e qualquer período da gestação, prestando-se um acompanhamento profissional adequado e que seja fator de proteção ao desenvolvimento e facilitador do processo de luto psíquico típico dessa situação.

Essa afirmativa é respaldada pelo resultado de que parte das gestantes percebeu como negativa a maneira como foi transmitida a notícia do diagnóstico, ressaltando a postura objetiva e fria do médico e, consequentemente, o impacto que isso resultou no enfrentamento da situação. Dessa forma, ressalta-se a importância da equipe de saúde estar atenta não somente aos fatores de risco físico, mas 
também psíquicos, os quais podem resultar de uma comunicação não eficiente e pouco acolhedora em condições de vulnerabilidade, como é o diagnóstico de malformação congênita.

Por outro lado, mais da metade das gestantes (55\%) percebeu como adequada a maneira como foi comunicada a notícia, fator importante que pode ter facilitado o processo de elaboração dos sentimentos iniciais de tristeza e medo para condições emocionais de confiança e tranquilidade, como observado nos relatos analisados. Isso também pode ter repercutido favoravelmente na percepção do companheiro que, posteriormente, passou a aceitar o diagnóstico e oferecer suporte à gestante na vivencia do choque, tristeza, autopiedade, culpa e até desespero, conforme ilustra o relato: "Ficou arrasado e muito preocupado, mas tentando me passar segurança".

Logo, é importante considerar que o diagnóstico de malformação congênita é um fator de risco para a saúde mental materna, podendo trazer consequências para o casal ao longo da gestação e repercutir mesmo depois do nascimento da criança, indicando a necessidade de intervenções voltadas tanto para a gestante quanto para sua rede de apoio, companheiro e demais familiares. Nesse sentido, a comunicação do diagnóstico, bem como sua percepção e compreensão, possui papel central na ressignificação da experiência de gestar um bebê malformado. Assim, conclui-se que, quando a notícia é transmitida de forma acolhedora, eficiente, com linguagem clara e informações objetivas e acessíveis, o impacto do diagnóstico pode ser minimizado, restabelecendo-se a confiança no acompanhamento médico, a aproximação e fortalecimento do casal, uma melhor aceitação familiar e, consequentemente, a elaboração de sentimentos e expectativas negativas os quais repercutem desfavoravelmente na saúde mental da mulher.

\section{Colaboradores}

A.C.B. CUNHA participou das etapas de concepção e desenho do estudo, coleta e análise de dados, discussão dos resultados, elaboração e redação final do concepção e desenho do estudo, coleta de dados e discussão dos resultados; C.L.V. CALDEIRA e V.M.S.P. CARNEIRO participaram das etapas de análise de dados, discussão dos resultados, elaboração e redação final do artigo.

\section{Referências}

Antunes, M. S. C., \& Patrocínio, C. (2007). A malformação do bebê. Vivências psicológicas do casal. Psicologia, Saúde \& Doenças, 8(2), 239-252.

Aymé, S. (2005). As injustiças do nascimento. Lisboa: Liga Internacional para o Estudo e Apoio da Deficiência Mental.

Bardin, L. (1977). Análise de conteúdo. Lisboa: Edições 70.

Barros, V. C., Santos, J. F. C., Lima, L. A., Fonseca, D. L., \& Lovisi, M. L. (2013). Depressão e apoio social em gestantes de fetos com malformações atendidas em um hospital materno-infantil público de referência no Rio de Janeiro. Cadernos Saúde Coletiva, 21(4), 391-402.

Brasil. Ministério da Saúde. (2013a). Departamento de Informática do Sistema Único de Saúde. Recuperado em dezembro 13, 2013, de http://www2.datasus.gov. br/DATASUS/index.php/index.php?area=0201

Brasil. Presidência da República. (2013b). Decreto-Lei $n^{\circ} 2.848$, de 7 de dezembro de 1940. Código Penal. Recuperado em dezembro 13, 2013, de http://www. planalto.gov.br/ccivil_03/Decreto-Lei/Del2848.htm

Cardoso, J. A. (2001). Doenças genéticas: impacto psicológico e representações sociais. In M. Canavarro (Ed.), Psicologia da gravidez e da maternidade. Coimbra: Quarteto.

Chaplin, J. P., Schwitzer, R., \& Perkoulidis, S. A. (2005). Experiences of prenatal diagnosis of spina bifida or hydrocephalus in parents who decide to continue with their pregnancy. Journal of Genetic Counseling, 14(2), 151-162.

Cunha, J. A. (2001). Manual da versão em português das escalas Beck. São Paulo: Casa do Psicólogo.

Farias, L. N., \& Villwock, C. (2010). Luto na maternidade: a perda real. Recuperado em julho 22, 2013, de http:// guaiba.ulbra.tche.br/pesquisa/2010/artigos/psicologia/ salao/655.pdf

Gomes, A. G., \& Piccinini, C. A. (2010). Malformação no bebê e maternidade: aspectos teóricos e clínicos. Psicologia Clínica, 22(1),15-38.

Gorayeb, R. P. (2008). Intervenção psicológica com gestantes cujos fetos apresentam malformação congênita (Tese de doutorado não-publicada). Universidade de São Paulo, Ribeirão Preto. 
Ha, J. F., Anat, D. S., \& Longnecker, N. (2010). Doctorpatient communication: A review. The Ochsner Journal, 10(1), 38-43.

Leithner, K. (2011). The psychic state of the pregnant woman and prenatal diagnostic procedures. In D. Prayer (Ed.), Fetal MRI. Viena: Springer.

Loaiza, J. L., \& Arroyave, A. M. (2009). Deliveryng bad news: Aspects to consider when training medical students and residentes. Donald School Journal of Ultrasound and Obstetrics and Ginecology, 3(4), 65-68.

Montero, S. M. P., Sánchez, J. M. R., Montoro, C. H., Crespo, M. L., Jaén, A. G. V., \& Tirado, M. B. R. (2011). A experiência da perda perinatal a partir da perceptiva dos profissionais de saúde. Revista Latino-Americana de Enfermagem, 19(6), 1405-1412. Recuperado em julho 22, 2013, de http://www.scielo.br/pdf/rlae/ v19n6/pt_18.pdf

Perosa, G. B., Canavez, I. C., Silveira, F. C. P., Padovani, F. H. P., \& Peraçoli, J. C. (2009). Sintomas depressivos e ansiosos em mães de recém-nascidos com e sem malformações. Revista Brasileira de Ginecologia \& Obstetrícia, 31(9), 433-439.

Perosa, G. B., \& Ranzani, P. M. (2008). Capacitação do médico para dar más notícias à criança. Revista Brasileira de Educação Médica, 32(4), 468-473.

Ramos, T. C. L., Hoffmann, V. M. B., \& Regen, M. (1985). As dificuldades em transmitir a notícia: pesquisa junto a pais de pacientes portadores de Síndrome de Down. Revista Brasileira de Deficiência Mental, 18, 47-69.

Roecker, S., Mai, L. D., Baggio, S. C., Mazzola, J. C., \& Marcon, S. S. (2012). Vivência de mães de bebês com malformação. Escola Anna Nery, 16(1), 17-26.

Rosa, R., Martins, F. E., Gasperi, B. L., Monticelli, M., Siebert, E. R. C., \& Martins, N. M. (2010). Mãe e filho: os primeiros laços de aproximação. Escola Anna Nery, 14(1), 105-112.

Sarmento, R., \& Setúbal, M. R. V. (2003). Abordagem psicológica em obstetrícia: aspectos emocionais da gravidez, parto e puerpério. Revista Ciências Médicas, 12(3), 261-268.

Setúbal, M. S., Barini, R., Zaccaria, R., \& Silva, J. (2004). Reações psicológicas diante da gravidez complicada por uma malformação fetal. Recuperado em julho 22, 2013, de http://www.barini.med.br/publicacao

Soulé, M. (1992). Le psychiatre dans un service de medicine fetale et diagnostic anténatal. In M. Soulé, F. Begoin-Guignard, H. Cohen, F. Daffos, D. David, P. Faure, ... B. Schaal (Eds.), Introductionà la psychiatrie fetale. Les aspects psychologiques et psycopathologiques en medicinefetale et diagnostic prénatal. Paris: ESF.

Sousa, E. N., \& Muza, J. C. (2011). Quando a morte visita a maternidade: papel do psicólogo hospitalar no atendimento ao luto perinatal (Monografia não-publicada). Universidade Católica de Brasília.

Sousa, S. (2003). A saúde do feto. In E. Sá (Ed.), Psicologia do feto e do bebé. Lisboa: Fim de Século.

Sunelaitis, R. C., Arruda, D. C., \& Marcon, S. S. (2007) A repercussão de um diagnóstico de síndrome de Down no cotidiano familiar: perspectiva da mãe. Acta Paulista Enfermagem, 20(3) 264-271.

Vasconcelos, L., \& Petean, E. B. L. (2009). O impacto da malformação fetal: indicadores afetivos e estratégias de enfrentamento das gestantes. Psicologia, Saúde \& Doenças, 10(1), 69-82.

Recebido: dezembro 12, 2013

Versão final: setembro 2, 2014

Aprovado: setembro 19, 2014 
